Schlaganfallprophylaxe

\title{
Weltweite Patientencharta gibt Vorhofflimmern-Patienten eine Stimme
}

- Schlaganfallgefährdeten Patienten mit Vorhofflimmern (VF) vermeidbares Leid zu ersparen, ist das zentrale Anliegen einer von zahlreichen Fachgesellschaften und Patientenorganisationen getragenen Patientencharta. Das weltweite Projekt setzt vor allem auf eine bessere Aufklärung über die Risiken der häufigsten Herzrhythmusstörung.

Vorhofflimmern gilt nicht nur als eine der häufigsten Ursachen für einen Schlaganfall, sondern geht oft auch noch mit einer besonders schlechten Prognose einher. Durch Aufklärung und Sicherstellung präventiver Maßnahmen ließen sich nach Einschätzung von Dr. Markus Wagner, Stiftung Deutsche Schlaganfall-Hilfe, Gütersloh, um die $70 \%$ aller Schlaganfälle vermeiden. Unabdingbar ist in der Regel eine konsequente Antikoagulation.

Aufgrund einer geringen therapeutischen Breite gleicht die Antikoagulation mit herkömmlichen Vitamin-K-Antagonisten einer Fahrt zwischen "Skylla und Charybdis", sagte Prof. Erland Erdmann, Köln. Wegen der schwierigen Dosisfindung und einem nicht zu vernachlässigenden Interaktionspotenzial mit Medikamenten und Nahrungsmitteln sieht er Verbesserungsbedarf. Diesem werden moderne Antikoagulanzien wie der Faktor-Xa-Hemmer Rivaroxaban (Xarelto ${ }^{\circledR}$ ) offenbar gerecht. Erdmann konstatierte einen klaren „Fortschritt gegenüber älteren Medikamenten“.

\section{Fixe Dosierung ohne Routinekontrollen}

Im Zuge der ROCKED-AF-Studie erwies sich die einmal tägliche Gabe von $20 \mathrm{mg}$ Rivaroxaban gegenüber einem Vitamin-KAntagonisten als gleich effektiv. Hinsichtlich des Blutungsrisikos schnitt der FaktorXa-Hemmer tendenziell besser ab. Fatal verlaufende Blutungen traten signifikant

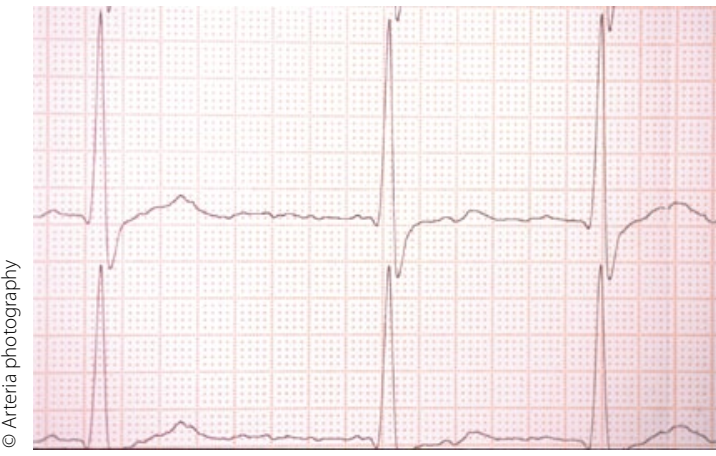

Vorhofflimmern: häufigste Ursache für einen Schlaganfall.

seltener auf, und die Inzidenz von Hirnblutungen war geringer. Die Tatsache, dass die Behandlung mit Rivaroxaban in fixer Dosierung (Dosisanpassung bei eingeschränkter Nierenfunktion) und ohne routinemäßige Laborkontrollen erfolgen kann, erleichtert Ärzten und Patienten das Leben.

- Dr. med. Ludger Riem

Quelle: Pressekonferenz „Rathausgespräche: Dialog Schlaganfall - Vorstellung der weltweiten Patienten-Charta,Sign Against Stroke in Atrial Fibrillation'", Düsseldorf-Benrath, Juni 2012 (Veranstalter: Bayer Vital)

\section{Chronische Obstipation}

\section{In Kürze wird S2k-Leitlinie publiziert}

- Chronische Obstipation verursacht gravierende Beschwerden und schränkt die Lebensqualität deutlich ein. Etwa 15\% der Bevölkerung leiden darunter, vor allem ältere Menschen. So sind Frauen jenseits des 65. Lebensjahres zu 26\%, Männer zu 16\% betroffen. Bei den über 84-Jährigen ist es knapp ein Drittel, bei Bewohnern von Pflegeheimen bis zu $80 \%$, erläuterte Prof. Martin Wehling, Heidelberg.

Der selektive Serotonin-5-HT4-Rezeptoragonist Prucaloprid (Resolor ${ }^{\oplus}$ ) lässt sich sicher und effektiv bei chronischer Obstipation einsetzen, auch bei älteren Menschen. In einer placebokontrollierten Untersuchung bei 300 über 65-jährigen Patienten erhöhte Prucaloprid nicht nur die Stuhlgangfrequenz, sondern reduzierte auch abdominelle Symptome und verbesserte die Lebensqualität. Es erwies sich auch in dieser Patientengruppe als sicher und gut verträglich. Zusätzlich zeigte eine Sicherheitsstudie an 89 älteren Patienten, dass es in Tagesdosen bis zu 2 mg nicht zu einer Verlängerung der QT-Zeit kommt.

\section{Therapie nach Stufenschema}

Der hohe Stellenwert von Prucaloprid in der Therapie der chronischen Obstipation schlägt sich nun auch in der interdisziplinär erstellten S2k-Leitlinie zur „Epidemiologie, Pathophysiologie, Diagnostik und Therapie der chronischen Obstipation" nieder, die unter Schirmherrschaft von DGNM (Deutsche Gesellschaft für Neurogastroenterologie und Motilität) und DGVS (Deutsche Gesellschaft für Verdauungs- und Stoffwechselkrankheiten) erstellt wurde und in Kürze publiziert werden soll. Danach sollte bei chronischer Obstipation als
Folge einer Transitstörung nach einem Stufenschema behandelt werden, beginnend mit Allgemeinmaßnahmen und der Einnahme von Ballaststoffen. An zweiter Stelle steht die medikamentöse Therapie mit osmotisch wirksamen Substanzen, bei Verträglichkeit auch mit Lactulose, Sorbitol sowie Bisacodyl und Natriumpicosulfat. Wird damit kein oder kein zufriedenstellender Erfolg erreicht, sollten laut Leitlinie Prokinetika vom Typ der 5-HT4-Agonisten wie Prucaloprid zum Einsatz kommen.

- Dr. Beate Fessler

Quelle: Satellitensymposium "Chronische Obstipation aus verschiedenen Blickwinkeln", Jahrestagung der Deutschen Gesellschaft für Verdauungs- und Stoffwechselkrankheiten, Hamburg, September 2012 (Veranstalter: Shire Deutschland) 